\title{
Mild Stimulation versus Conventional IVF: A Cost-Effectiveness Evaluation
}

\author{
Natalie M. Crawford ${ }^{1 *}$, Kashika M. Sahay ${ }^{2}$, Jennifer E. Mersereau ${ }^{1}$ \\ ${ }^{1}$ Department of Obstetrics and Gynecology, University of North Carolina, Chapel Hill, North Carolina, USA \\ ${ }^{2}$ Maternal and Child Heath University of North Carolina, Chapel Hill, North Carolina, USA \\ Email: "natalie_crawford@med.unc.edu, jennifer_mersereau@med.unc.edu, kmsahay@live.unc.edu
}

Received 29 October 2015; accepted 28 February 2016; published 2 March 2016

Copyright () 2016 by authors and Scientific Research Publishing Inc.

This work is licensed under the Creative Commons Attribution International License (CC BY).

http://creativecommons.org/licenses/by/4.0/

(c) (i) Open Access

\begin{abstract}
Purpose: To compare the strategy for mild stimulation IVF versus conventional stimulation IVF. Methods: A decision tree analytic model was created to compare IVF with mild stimulation versus convention stimulation in infertile women $<38$ years of age to evaluate which strategy is the least costly per live birth. Results: Using base-case estimates of costs and probabilities in women $<38$ years old with unexplained infertility, the cumulative live birth rate in the mild versus conventional stimulation group was $15.8 \%$ versus $28.6 \%$, respectively. The average cost for mild and convention IVF was $\$ 8789$ and $\$ 14,364$ per arm, respectively. In base case analysis, the ICER was $\$ 43,516$ representing the additional cost per live birth with conventional IVF. One-way sensitivity analysis evaluated the robustness of the data and revealed a tradeoff in which mild IVF stimulation had lower total costs and also lower live births versus conventional stimulation. Conclusions: Mild stimulation IVF has a lower cost per cycle but is also associated with lower live birth rates. Patient care decisions should be individualized irrespective of cost.
\end{abstract}

\section{Keywords}

Minimal Stimulation, Mild IVF, IVF, Decision Analysis, Cost Analysis

\section{Introduction}

The introduction of in vitro fertilization (IVF) dramatically improved the ability to help women with unexplained infertility achieve a pregnancy [1] [2]. Minimal or mild stimulation protocols for IVF (mild-IVF) have been developed to achieve a less vigorous ovarian response as compared to conventional stimulations protocols

"Corresponding author.

How to cite this paper: Crawford, N.M., Sahay, K.M. and Mersereau, J.E. (2016) Mild Stimulation versus Conventional IVF: A Cost-Effectiveness Evaluation. Open Journal of Obstetrics and Gynecology, 6, 180-188. 
(conv-IVF) [3] [4]. Many different approaches of mild stimulation exist, including use of oral agents such as clomiphene citrate or aromatase inhibitors, low-dose exogenous gonadotropins, gonadotropin releasing hormone $(\mathrm{GnRH})$ antagonists, and late follicular phase human chorionic gonadotropin (HCG) injection or lutenizing hormone (LH).

Mild ovarian stimulation has potential advantages over conv-IVF in select patient populations. Due to decreases in follicular number and retrieved oocytes, mild-IVF has been associated with a decrease in the risk of OHSS and multiple pregnancy rates [5]-[10]. Further, the less pronounced hormonal elevations seen with mildIVF may also be associated with an improvement in endometrial receptivity, potentially less risk of aneuploidy, and an improvement in obstetrical outcomes [11]-[13]. Patient preference is also important, as the psychological demands and the total cost per cycle appear to be less with mild-IVF as compared to conv-IVF [5] [14]-[16].

Mild-IVF may reduce the risk of complications seen with IVF, but an important concern with the use of a mild stimulation protocol is the potential reduction of pregnancy rates [4] [17]. Mild-IVF usually results in a lower total dose of exogenous gonadotropins, fewer clinic visits, fewer oocytes retrieved, and fewer embryos [5] [7]. A decrease in embryo number may lower fresh IVF pregnancy rates, diminish the opportunity for cryopreservation of extra embryos and future frozen embryo transfer (FET), and may lead to reduction in cumulative live birth rate (LBR) [8] [18].

Although European studies have concluded that mild-IVF was cost-effective over conv-IVF when medications were minimized, no such comparison of mild-IVF and conv-IVF has been undertaken in the United States (US) [16] [19] [20]. As both costs and expected pregnancy rates in the United States are, on average, higher than reported European rates, application of a European assessment on cost-effectiveness is difficult in the US [21]-[23]. Therefore, we performed a decision analysis to determine the cost per live birth in women $<38$ years of age with unexplained infertility who undergo mild-IVF versus those who undergo conv-IVF.

\section{Methods and Materials}

A decision tree analysis model was developed comparing two strategies, mild-IVF and conv-IVF in infertile women under age 38 years with either unexplained, male or tubal factor infertility in order to determine the cost per live birth. The model was structured using the Consolidated Health Economic Evaluation Reporting Standards (CHEERS) as a framework [24]. The model (Figure 1) was designed so that with each cycle, the following possibilities may occur: 1) there will be enough embryos to transfer after fresh mild-IVF without additional embryos for cryopreservation; 2) there will be enough embryos to transfer after fresh conv-IVF with additional embryos for cryopreservation; and 3) there will be enough embryos to transfer after fresh conv-IVF without additional embryos for cryopreservation. If pregnancy does not occur, the conv-IVF model allows for a total of one fresh and one frozen cycle to try to achieve a pregnancy. The base case cohort size was 1000 women. Our local Institutional Review Board does not require approval for decision analysis project.

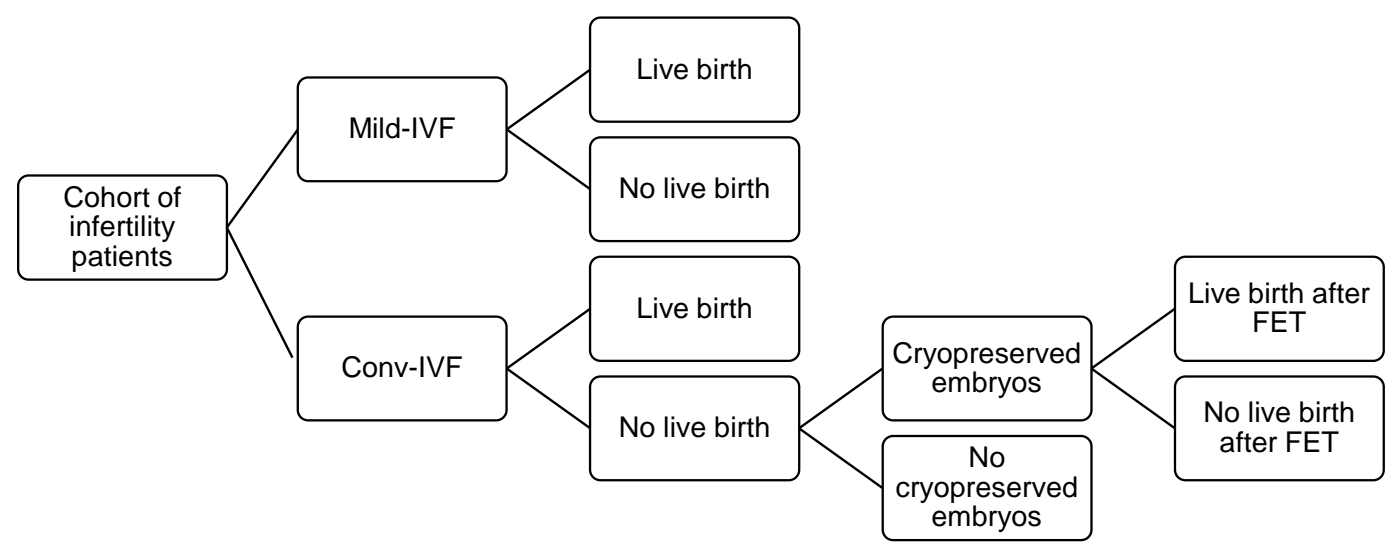

Figure 1. Simplified decision tree for comparing mild and conventional stimulation for IVF in a cohort of women with unexplained infertility. In this diagram, a square represents a decision node and a circle represent a chance node. In the mild-IVF arm, women go through one fresh cycle. Women in the conv-IVF arm may undergo one fresh and one frozen embryo transfer if embryos were available for cryopreservation. 
Our probability inputs for live birth per fresh cycle and cycle cancellation were based on the largest randomized controlled trial comparing conv-IVF and mild-IVF [5]. In this Netherlands-based study by Heijen et al, participants were $<38$ years of age with unexplained, male or tubal factor infertility. 404 subjects were randomized to either conv-IVF or mild-IVF. The mild-IVF protocol included GnRH antagonist treatment, 150 IU FSH daily starting on cycle day 5, and transfer of a single embryo while the conv-IVF protocol included GnRH agonist treatment, 150 IU FSH daily starting on cycle day 2, and a double embryo transfer [5] [25]. The mean duration of gonadotropin stimulation and number of oocytes retrieved was 8.3 days and 6.9 oocytes (SD 4.8) for mild-IVF and 11.5 days and 8.5 oocytes (SD 4.3) for conv-IVF [5]. The mean total gonadotropin usage was 1307 IU for mild-IVF versus 1832 IU for conv-IVF [5]. Specifically, the LBR per fresh cycle for mild-IVF versus conv-IVF was reported at $15.8 \%$ versus $24 \%$, respectively (Table 1 ). Cycle cancellation rates were $18 \%$ for mild-IVF and 8.3\% for conv-IVF; however, as input probabilities were based on LBR per cycle, cycle cancellation probabilities were not included as inputs in the model [5].

To estimate base case inputs for supernumery embryo cryopreservation and LBR after frozen embryo transfer, the previously discussed study by Heijen et al. had only a few patients with extra embryos for cryopreservation and a low LBR per FET (1.1\%) [5]. As we felt this is not representative of expected success rate after FET, we opted to base LBRs after FET on a large Swedish study by Thurin et al. [26]. In this study, subjects received a conv-IVF stimulation protocol and were randomized to either double fresh embryo transfer or single fresh embryo transfer with a subsequent single-embryo FET. The LBR per FET was 16.4\% [26]. Furthermore, we assumed not all patients who underwent conv-IVF would have extra embryos available for cryopreservation. The probability input for cryopreservation of embryos is from a Netherlands based study in which the probability of having excess embryos for cryopreservation after conv-IVF was 37\% [16]. In our model, patients pursuing mild-IVF were assumed to not have additional embryos available for cryopreservation or FET. Of note, due to a lack of randomized controlled data from the US on mild-IVF, we based all probability inputs on European studies in order to achieve a cohesive cohort for analysis.

Cost was interpreted from a patient directed medical cost perspective (Table 1). Total costs for a fresh cycle included both medication and procedural costs. We estimated medication costs for both protocols based on an average of costs from known US fertility pharmacies, with an average follicle stimulating hormone (FSH) cost at \$0.91 per IU, an average cost for 5 days of GnRH antagonist at \$573, and an average cost for a GnRH agonist kit at \$149 [27] [28]. We calculated both a total FSH cost and a total stimulation medication costs for mild-IVF and conv-IVF protocols. We estimated procedural IVF costs for conv-IVF (facility, monitoring, procedural and laboratory costs), cryopreservation costs, and FET costs by taking an average of seven different IVF centers in New York, Massachusetts, North Carolina, Texas, Colorado, Illinois, California [29]-[35]. Similarly, for mildIVF, we took the average of six US clinics that advertise mild-IVF procedural costs (facility, monitoring, procedural and laboratory costs) from New York, North Carolina, Texas, Utah and California [35]-[40]. Total costs for a fresh cycle included both medication and procedural costs. Loss of productivity, obstetrical costs, and indirect costs were not included in this comparison.

The incremental cost-effectiveness ratio (ICER) represents the additional cost per live birth. The ICER was calculated by dividing the cost difference between the two scenarios by live birth difference. A protocol was considered dominated when it was equally or less effective than the alternative but more costly. The robustness of the base-case results was tested using one-way sensitivity analysis by varying probabilities and costs down to one-half and up to two times the baseline estimates (Table 1). In addition, Monte Carlo analysis was performed in which all probability estimates were varied simultaneously and the simulation was run 62,718 cycles (the total number of IVF cycles performed in the US in 2012 in this age group) [23]. The model was built in Microsoft Excel 2013, with the add-in macro Oracle Crystal Ball (version 11.1.2.2) for Monte Carlo simulation.

\section{Results}

In our analytic model for women $<38$ years old with unexplained infertility, the cumulative LBR with mild-IVF versus conv-IVF was $15.8 \%$ and $28.6 \%$, respectively (Table 2 ). The average cost per woman was $\$ 8789$ and $\$ 14,364$ for the mild-IVF and the conv-IVF arms, respectively. Thus, the mild-IVF arm had a lower percentage of live births and lower total costs. As the conv-IVF arm had a 12.8\% higher LBR than mild-IVF, the ICER was $\$ 43,516$, representing the additional cost per live birth with conv-IVF.

Using one-way sensitivity analysis for each input variable, the model was sensitive to changes in LBR after IVF (Table 3). When varying the effectiveness (LBR) of mild-IVF and conv-IVF, in most scenarios there is a 
Table 1. Probability and cost variables.

\begin{tabular}{|c|c|c|c|}
\hline & Base case & Ranges & Reference \\
\hline \multicolumn{4}{|l|}{ Probability variables } \\
\hline \multicolumn{4}{|l|}{ Mild-IVF } \\
\hline Cycle cancelation & 0.180 & $0.090-0.360$ & [5] \\
\hline Live birth per cycle & 0.158 & $0.079-0.316$ & [5] \\
\hline \multicolumn{4}{|l|}{ Conv-IVF } \\
\hline Cycle cancelation & 0.083 & $0.042-0.166$ & [5] \\
\hline Live birth per cycle & 0.240 & $0.120-0.480$ & [5] \\
\hline Cryopreserved embryos & 0.370 & $0.185-0.740$ & [15] \\
\hline Live birth after FET & 0.164 & $0.082-0.328$ & [25] \\
\hline \multicolumn{4}{|l|}{$\underline{\text { Cost variables }}^{1}$} \\
\hline \multicolumn{4}{|l|}{ Mild-IVF } \\
\hline Total FSH cost ${ }^{2}$ & 1189 & $595-2378$ & [5] [26] [27] \\
\hline Total medication $\operatorname{cost}^{3}$ & 2399 & $1200-4798$ & [5] [26] [27] \\
\hline Fresh transfer cycle ${ }^{4}$ & 8789 & $4395-17,578$ & [5] [26] [27] [34]-[39] \\
\hline \multicolumn{4}{|l|}{ Conv-IVF } \\
\hline Total FSH $\operatorname{cost}^{2}$ & 1667 & $834-3334$ & [5] [26] [27] \\
\hline Total medication cost ${ }^{3}$ & 2601 & $1301-5202$ & [5] [26] [27] \\
\hline Fresh transfer cycle ${ }^{4}$ & 12,991 & $6496-25,982$ & [5] [26]-[34] \\
\hline Cryopreservation and storage & 1150 & $575-2300$ & [28]-[34] \\
\hline FET cycle & 3370 & $1685-6740$ & [28]-[34] \\
\hline
\end{tabular}

${ }^{1}$ All cost variables reported in 2015 USD; ${ }^{2}$ Calculated from average cost per IU FSH; ${ }^{3}$ Total cost of GnRH agonist or GnRH antagonist, FSH, HCG and progesterone; ${ }^{4}$ Total cost including all medications, facility, and lab costs.

Table 2. Base case analysis results.

\begin{tabular}{cccccc} 
& Cost $^{1}$ & Incremental cost $^{1}$ & Effectiveness $^{2}$ & Incremental effectiveness $^{2}$ & ICER $^{3}$ \\
\hline Mild-IVF & 8789 & & 15.8 & & \\
Conv-IVF & 14,364 & 5575 & 28.6 & 12.8 & 43,516 \\
\hline
\end{tabular}

Note: based on a simulated cohort of 1000 women; ${ }^{1}$ Cost reported as average cost per woman in 2015 USD; ${ }^{2}$ Effectiveness reported as \% live births; ${ }^{3} \mathrm{ICER}=$ Incremental cost-effectiveness ratio.

Table 3. One-way sensitivity analysis results.

\begin{tabular}{|c|c|c|c|c|c|}
\hline & Value & Incremental cost ${ }^{1}$ & Incremental effectiveness $^{2}$ & $\mathrm{ICER}^{3}$ & Interpretation $^{4}$ \\
\hline \multicolumn{6}{|c|}{ Effectiveness } \\
\hline \multicolumn{6}{|c|}{ Mild-IVF } \\
\hline Low & 0.07 & (5575) & $(20.7)$ & 26,918 & tradeoff \\
\hline High & 0.32 & (5575) & 3.0 & $(186,564)$ & mild-IVF \\
\hline \multicolumn{6}{|c|}{ Conv-IVF } \\
\hline Low & 0.12 & (5276) & (1.5) & 371,777 & tradeoff \\
\hline High & 0.48 & $(1347)$ & $(35.4)$ & 14,922 & tradeoff \\
\hline \multicolumn{6}{|l|}{ Cost } \\
\hline \multicolumn{6}{|c|}{ Mild-IVF } \\
\hline Low & 4395 & (9969) & $(12.8)$ & 77,813 & tradeoff \\
\hline High & 17,578 & 3214 & $(12.8)$ & & conv-IVF \\
\hline \multicolumn{6}{|c|}{ Conv-IVF } \\
\hline Low & 6496 & 1902 & $(12.8)$ & & conv-IVF \\
\hline High & 25,982 & (9709) & $(12.8)$ & 75,781 & tradeoff \\
\hline
\end{tabular}

Note: based on a simulated cohort of 1000 women. ${ }^{1}$ Cost reported as average cost per woman in 2015 USD, negative values in parenthesis; ${ }^{2}$ Effectiveness reported as \% live births, negative values in parenthesis; ${ }^{3}$ ICER $=$ Incremental cost-effectiveness ratio; ${ }^{4}$ Interpretation reported as tradeoff (mild-IVF with lower costs but lower effectiveness), mild-IVF (mild-IVF is cost-effective), or conv-IVF (mild-IVF is not cost-effective). 
tradeoff with mild-IVF-costs are lower but LBRs are lower as well. In the scenario where mild-IVF is at maximum hypothetical effectiveness (32\% LBR), mild-IVF is cost-effective over conv-IVF. When varying the costs for mild-IVF and conv-IVF, conv-IVF dominated mild-IVF when either mild-IVF is at maximum cost or convIVF is at minimum cost. In the other scenarios, mild-IVF was again a tradeoff with lower costs and lower LBRs.

Using Monte Carlo simulation with 62,718 cycles over 1000 trials, mild-IVF resulted in a median of 9623 fewer live births and the median ICER was \$37,956 additional cost per live birth with conv-IVF. In the simulation, mild-IVF had lower costs and fewer live births in over $90 \%$ of scenarios.

\section{Discussion}

Our study is the first to use an analytic decision model to compare the cost per live birth using US cost-estimates for mild-IVF and conv-IVF. Our base-case and most sensitivity analyses resulted in a tradeoff in which mildIVF has a lower cost and a lower effectiveness than conv-IVF. The one scenario which found mild-IVF to be cost effective required mild-IVF to be at maximum hypothetical effectiveness (32\%). This should be cautiously interpreted and is a highly unlikely scenario, as mild-IVF would need to be more have higher LBRs than convIVF (32\% versus 24\%, respectively) in order to be cost effective. Further, the two scenarios where conv-IVF dominated mild-IVF are also unlikely, requiring mild-IVF to have a greater total cost than conv-IVF. The Monte Carlo simulation further confirmed the tradeoff with mild-IVF resulting in lower costs but lower effectiveness in the vast majority of scenarios.

Previous cost-effectiveness evaluations comparing mild-IVF and conv-IVF have been limited to only a European perspective. Polinder et al. evaluated subjects randomized to either mild-IVF or conv-IVF with the outcome of cumulative 1 year LBRs after multiple cycles [20]. In this analysis, patients who underwent mild-IVF had an increase in total number of cycles completed throughout the year, and mild-IVF was determined to be a cost-effective alternative to conv-IVF [20]. A main contributing factor to the lower cost for mild-IVF was the inclusion of obstetrical and neonatal costs associated with multiple gestations in the conv-IVF group [20]. Further, Groen et al. performed a cost-effectiveness evaluation after retrospective review of patients who received mild-IVF or conv-IVF [16]. Depending on the number of cycles per patient, mild-IVF was at times cost-effective when medications were minimized [16]. However, as patients were not randomized in this study, selection bias may exist in which subjects were allocated a treatment option in a preferential fashion, thus skewing the results [16]. Due to these limitations, and as there are substantial differences in costs between Europe and the US, it is difficult to apply these results to clinical practice in the US.

As with all decision analytic models, the strength of our conclusion is dependent upon the validity of our data inputs. In our model, the base case inputs for live birth per fresh cycle, excess embryos for cryopreservation, and frozen embryo transfer live birth rates are all based on European studies in the same era in order to achieve a cohesive cohort for analysis. We used the largest published randomized control trial as the base for LBRs after fresh mild-IVF and conv-IVF cycles [5]. As this study is randomized, variability and provider initiated selection bias between treatment group assignment was minimized, thus improving validity. Further, LBRs in this study (15.8\% for mild-IVF) are consistent with the LBR for mild-IVF reported in another randomized European study by Hohmann et al. (16\%), supporting the validity of these results [5] [41]. However, LBRs in these European studies are lower than expected US rates [5] [16] [23] [26]. If our model compared the LBR from Heijen et al. for mild-IVF with US data for live birth per cycle in women $<38$ years, conv-IVF would dominate the model due to markedly higher US LBRs [5] [23] [42]. Therefore, because IVF practices and success rates differ between Europe and the US, we felt that the strongest model included LBRs for mild-IVF, conv-IVF, and FET based on European studies, as this would give us the most direct comparison. Our sensitivity analysis allowed for further broad analysis of the varying LBRs to better include scenarios that may approximate US outcomes.

Our model focused solely on live birth, we did not include differences in the potential for multiple gestation, potential losses in productivity, or the emotional burden associated with infertility treatment. Multiple gestation has a significant impact on obstetrical and neonatal costs, and inclusion of this factor in our model would significantly increase the cost of conv-IVF [43]-[45]. However, with the gaining acceptance of elective single embryo transfer in the US, the transfer of a single embryo, regardless of stimulation protocol, is likely the most direct way to decrease the risk of multiple gestation [46]-[48].

Unfortunately, there is not published US data on the cost for mild-IVF. European studies that report a break- 
down for the cost of mild-IVF and conv-IVF have very similar monitoring and procedural costs [5] [16] [20]. However, many US infertility practices advertise decreased costs for "mild”, "mini”, or "minimal” IVF as compared to conv-IVF. In our model, we opted to use an average of advertised costs in order to most closely reflect the difference in monitoring charges with a mild versus conventional stimulation approach. Regional bias was minimized by averaging IVF rates for both mild-IVF and conv-IVF from clinics across the US and sensitivity analysis allowed for further broad analysis of the costs. The total costs in our model for conv-IVF (procedural and medication costs) and FET were similar to previously reported US rates [21] [43] [49]-[51].

The difference in conv-IVF stimulation protocols between European studies and conventional US practice may impact interpretation of our model. In the reported base-case study, the FSH dose in conv-IVF was limited to 150 IU per day (average 1832 IU per cycle) [5] [41]. This is lower than what is reported in US studies, and may have an impact on the degree of stimulation, number of oocytes retrieved, and total costs. A surveillance report in the US revealed a mean FSH dose per GnRH agonist cycle of 2186 IU [52]. Although this is higher than the mean FSH dose in our conv-IVF group, the overall FSH cost difference is limited (\$322 USD) [5] [52]. Thus, we would not expect an increase in FSH dose in conventional US protocols to make a substantial difference in the results of our model. Interestingly, the overall medication cost in our model was not markedly different for the mild-IVF and conv-IVF groups. This is likely due to the balance between the cost difference in total FSH dose per protocol and the price difference between GnRH agonist and antagonist treatment. Although the total length of stimulation and total FSH dose is less in mild-IVF, GnRH antagonist is more expensive than agonist treatment [5] [27] [28] [53]. It is important to note that our results apply to the mild-IVF protocol used in our model, which includes GnRH antagonist treatment and exogenous gonadotropins. Other ovarian stimulation protocols that avoid the use gonadotropins (such as natural cycle or oral agents for ovarian stimulation), would be expected to result in a decreased cost and lower LBRs and are not included in the scope of the analysis.

\section{Conclusion}

Mild stimulation protocols have lower costs and also lower LBRs when compared with conventional stimulation. Although mild stimulation may be advantageous for select patients, mild-IVF should not be promoted over conv-IVF on the basis of cost-saving without disclosing a decrease in LBRs. Decisions regarding the appropriate stimulation plan should be individualized for the patient based on clinical reasoning and patient preferences. Additional randomized control trials in the US comparing mild-IVF and conv-IVF may help clarify the potential benefits of a mild stimulation protocol.

\section{Conflict of Interest}

The authors declare that they have no conflict of interest.

\section{References}

[1] Practice Committee of the American Society for Reproductive Medicine (2006) Effectiveness and Treatment for Unexplained Infertility. Fertility and Sterility, 86, S111-S114. http://dx.doi.org/10.1016/j.fertnstert.2006.07.1475

[2] Pandian, Z., Gibreel, A. and Bhattacharya, S. (2012) In Vitro Fertilisation for Unexplained Subfertility. The Cochrane Database of Systematic Reviews, 4, Published Online. http://dx.doi.org/10.1002/14651858.cd003357.pub3

[3] Nargund, G., Fauser, B.C., Macklon, N.S., Ombelet, W., Nygren, K. and Frydman, R. (2007) The ISMAAR Proposal on Terminology for Ovarian Stimulation for IVF. Human Reproduction, 22, 2801-2804. http://dx.doi.org/10.1093/humrep/dem285

[4] Fauser, B.C., Nargund, G., Andersen, A.N., Norman, R., Tarlatzis, B., Boivin, J., et al. (2010) Mild Ovarian Stimulation for IVF: 10 Years Later. Human Reproduction, 25, 2678-2684. http://dx.doi.org/10.1093/humrep/deq247

[5] Heijnen, E.M., Eijkemans, M.J., De Klerk, C., Polinder, S., Beckers, N.G., Klinkert, E.R., et al. (2007) A Mild Treatment Strategy for In-Vitro Fertilisation: A Randomised Non-Inferiority Trial. Lancet, 369, 743-749. http://dx.doi.org/10.1016/S0140-6736(07)60360-2

[6] Steward, R.G., Lan, L., Shah, A.A., Yeh, J.S., Price, T.M., Goldfarb, J.M., et al. (2014) Oocyte Number as a Predictor for Ovarian Hyperstimulation Syndrome and Live Birth: An Analysis of 256,381 in Vitro Fertilization Cycles. Fertility and Sterility, 10, 967-973. http://dx.doi.org/10.1016/j.fertnstert.2013.12.026

[7] Verberg, M.F., Eijkemans, M.J., Macklon, N.S., Heijnen, E.M., Baart, E.B., Hohmann, F.P., et al. (2009) The Clinical Significance of the Retrieval of a Low Number of Oocytes Following Mild Ovarian Stimulation for IVF: A Meta- 
Analysis. Human Reproduction Update, 15, 5-12. http://dx.doi.org/10.1093/humupd/dmn053

[8] Allersma, T., Farquhar, C. and Cantineau, A.E. (2013) Natural Cycle in Vitro Fertilisation (IVF) for Subfertile Couples. The Cochrane Database of Systematic Reviews, 8, Cd010550. http://dx.doi.org/10.1002/14651858.cd010550.pub2

[9] Bensdorp, A.J., Tjon-Kon-Fat, R.I., Bossuyt, P.M., Koks, C.A., Oosterhuis, G.J., Hoek, A., et al. (2015) Prevention of Multiple Pregnancies in Couples with Unexplained or Mild Male Subfertility: Randomised Controlled Trial of in Vitro Fertilisation with Single Embryo Transfer or in Vitro Fertilisation in Modified Natural Cycle Compared with Intrauterine Insemination with Controlled Ovarian Hyperstimulation. BMJ, 350, g7771. http://dx.doi.org/10.1136/bmj.g7771

[10] Casano, S., Guidetti, D., Patriarca, A., Pittatore, G., Gennarelli, G. and Revelli, A. (2012) MILD Ovarian Stimulation with GnRH-Antagonist vs. Long Protocol with Low Dose FSH for Non-PCO High Responders Undergoing IVF: A Prospective, Randomized Study Including Thawing Cycles. Journal of Assisted Reproduction and Genetics, 29, 1343-1351. http://dx.doi.org/10.1007/s10815-012-9863-2

[11] Santos, M.A., Kuijk, E.W. and Macklon, N.S. (2010) The Impact of Ovarian Stimulation for IVF on the Developing Embryo. Reproduction, 139, 23-34. http://dx.doi.org/10.1530/REP-09-0187

[12] Baart, E.B., Martini, E., Eijkemans, M.J., Van Opstal, D., Beckers, N.G., Verhoeff, A., et al. (2007) Milder Ovarian Stimulation for In-Vitro Fertilization Reduces Aneuploidy in the Human Preimplantation Embryo: A Randomized Controlled Trial. Human Reproduction, 22, 980-988. http://dx.doi.org/10.1093/humrep/del484

[13] Pelinck, M.J., Keizer, M.H., Hoek, A., Simons, A.H., Schelling, K., Middelburg, K., et al. (2010) Perinatal Outcome in Singletons after Modified Natural Cycle IVF and Standard IVF with Ovarian Stimulation. European Journal of Obstetrics, Gynecology, and Reproductive Biology, 148, 56-61. http://dx.doi.org/10.1016/j.ejogrb.2009.09.015

[14] de Klerk, C., Heijnen, E.M., Macklon, N.S., Duivenvoorden, H.J., Fauser, B.C., Passchier, J., et al. (2006) The Psychological Impact of Mild Ovarian Stimulation Combined with Single Embryo Transfer Compared with Conventional IVF. Human Reproduction, 21, 721-727. http://dx.doi.org/10.1093/humrep/dei395

[15] de Klerk, C., Macklon, N.S., Heijnen, E.M., Eijkemans, M.J., Fauser, B.C., Passchier, J., et al. (2007) The Psychological Impact of IVF Failure after Two or More Cycles of IVF with a Mild versus Standard Treatment Strategy. Human Reproduction, 22, 2554-2558. http://dx.doi.org/10.1093/humrep/dem171

[16] Groen, H., Tonch, N., Simons, A.H., van der Veen, F., Hoek, A. and Land, J.A. (2013) Modified Natural Cycle versus Controlled Ovarian Hyperstimulation IVF: A Cost-Effectiveness Evaluation of Three Simulated Treatment Scenarios. Human Reproduction, 28, 3236-3246. http://dx.doi.org/10.1093/humrep/det386

[17] Verberg, M.F., Macklon, N.S., Nargund, G., Frydman, R., Devroey, P., Broekmans, F.J., et al. (2009) Mild Ovarian Stimulation for IVF. Human Reproduction Update, 15, 13-29. http://dx.doi.org/10.1093/humupd/dmn056

[18] Stanger, J.D. and Yovich, J.L. (2013) Follicle Recruitment Determines IVF Productivity Rate via the Number of Embryos Frozen and Subsequent Transfers. Reproductive Biomedicine Online, 27, 286-296. http://dx.doi.org/10.1016/j.rbmo.2013.05.015

[19] Lou, H.Y. and Huang, X.Y. (2010) Modified Natural Cycle for in Vitro Fertilization and Embryo Transfer in Normal Ovarian Responders. The Journal of International Medical Research, 38, 2070-2076. http://dx.doi.org/10.1177/147323001003800622

[20] Polinder, S., Heijnen, E.M., Macklon, N.S., Habbema, J.D., Fauser, B.J. and Eijkemans, M.J. (2008) Cost-Effectiveness of a Mild Compared with a Standard Strategy for IVF: A Randomized Comparison Using Cumulative Term Live Birth as the Primary Endpoint. Human Reproduction, 23, 316-323. http://dx.doi.org/10.1093/humrep/dem372

[21] Chambers, G.M., Sullivan, E.A., Ishihara, O., Chapman, M.G. and Adamson, G.D. (2009) The Economic Impact of Assisted Reproductive Technology: A Review of Selected Developed Countries. Fertility and Sterility, 91, 2281-2294. http://dx.doi.org/10.1016/j.fertnstert.2009.04.029

[22] Baker, V.L. (2013) Mild Ovarian Stimulation for in Vitro Fertilization: One Perspective from the USA. Journal of Assisted Reproduction and Genetics, 30, 197-202. http://dx.doi.org/10.1007/s10815-013-9946-8

[23] Centers for Disease Control and Prevention, American Society for Reproductive Medicine, Society for Assisted Reproductive Technology (2014) 2012 Assisted Reproductive Technology: Fertility Clinic Success Rates Report. Department of Health and Human Services, Centers for Disease Control and Prevention, Atlanta. http://www.cdc.gov/art/pdf/2012-report/art-2012-fertility-clinic-report.pdf

[24] Husereau, D., Drummond, M., Petrou, S., Carswell, C., Moher, D., Greenberg, D., et al. (2013) Consolidated Health Economic Evaluation Reporting Standards (CHEERS) Statement. International Journal of Technology Assessment in Health Care, 29, 117-122. http://dx.doi.org/10.1017/S0266462313000160

[25] Eijkemans, M.J., Heijnen, E.M., de Klerk, C., Habbema, J.D. and Fauser, B.C. (2006) Comparison of Different Treatment Strategies in IVF with Cumulative Live Birth over a Given Period of Time as the Primary End-Point: Methodological Considerations on a Randomized Controlled Non-Inferiority Trial. Human Reproduction, 21, 344-351. http://dx.doi.org/10.1093/humrep/dei332 
[26] Thurin, A., Hausken, J., Hillensjo, T., Jablonowska, B., Pinborg, A., Strandell, A., et al. (2004) Elective Single-Embryo Transfer versus Double-Embryo Transfer in in Vitro Fertilization. The New England Journal of Medicine, 351, 2392-2402. http://dx.doi.org/10.1056/NEJMoa041032

[27] Freedom Fertility Pharmacy. Accessed 27 January 2015. http://prices.freedomfertility.com

[28] MDR Specialty Pharmacy. Accessed 22 January 2015. http://www.mdrusa.com/price-list

[29] Estimated Cost for Infertility Treatment. Center for Assisted Reproduction, Bedford. Accessed 30 January 2015. http://www.embryo.net/fertility-cost/infertility-treatment-cost

[30] Single Cycle IVF Cost Details. Advanced Fertility Center of Chicago, Chicago. Accessed on 30 January 2015. http://www.advancedfertility.com/ivfprice.htm

[31] IVF Assist. Fertility Centers of New England, Reading. Accessed on 30 January 2015. http://www.fertilitycenter.com/affording_treatment/ivf_assist

[32] Cash Discounted Fees. San Diego Fertility Center, San Diego. Accessed on 30 January 2015. http://www.sdfertility.com/ivfcashoption.htm

[33] New York University Fertility Canter, New York. Accessed on 30 January 2015. http://www.nyufertilitycenter.org/financial_information

[34] Colorado Center for Reproductive Medicine. IVF Treatment Costs. Accessed on 30 January 2015. https://www.ccrmivf.com/colorado/treatment-costs/

[35] Piedmont Reproductive Endocrinology. Minimal IVF. Accessed on 30 January 2015. http://www.pregonline.com/minimal_ivf.php

[36] IVF MD, Dallas. Accessed on 4 February 2015. http://www.ivfmd.net/about-ivfmd/cost-of-mini-ivf

[37] Neway Fertility, New York. Accessed on 4 February 2015. http://newayfertility.com/fertility-services/

[38] Alta Bates in Vitro Fertilization Program, Berkeley. Accessed on 4 February 2015. http://www.abivf.com/

[39] Reproductive Fertility, Los Angeles. Accessed on 4 February 2015. http://www.reproductivefertility.com/packages.html

[40] East Bay Fertility Center, Provo. Accessed on 4 February 2015. http://www.utahinfertility.com/pricing/

[41] Hohmann, F.P., Macklon, N.S. and Fauser, B.C. (2003) A Randomized Comparison of Two Ovarian Stimulation Protocols with Gonadotropin-Releasing Hormone (GnRH) Antagonist Cotreatment for in Vitro Fertilization Commencing Recombinant Follicle-Stimulating Hormone on Cycle Day 2 or 5 with the Standard Long GnRH Agonist Protocol. The Journal of Clinical Endocrinology and Metabolism, 88, 166-173. http://dx.doi.org/10.1210/jc.2002-020788

[42] Centers for Disease Control and Prevention, American Society for Reproductive Medicine, Society for Assisted Reproductive Technology (2008) 2006 Assisted Reproductive Technology Success Rates: National Summary and Fertility Clinics Report. Department of Health and Human Services, Centers for Disease Control and Prevention, Atlanta. http://www.cdc.gov/art/pdf/archived/2006art.pdf

[43] Little, S.E., Ratcliffe, J. and Caughey, A.B. (2006) Cost of Transferring One through Five Embryos per in Vitro Fertilization Cycle from Various Payor Perspectives. Obstetrics and Gynecology, 108, 593-601. http://dx.doi.org/10.1097/01.AOG.0000230534.54078.b3

[44] Callahan, T.L., Hall, J.E., Ettner, S.L., Christiansen, C.L., Greene, M.F. and Crowley Jr., W.F. (1994) The Economic Impact of Multiple-Gestation Pregnancies and the Contribution of Assisted-Reproduction Techniques to Their Incidence. The New England Journal of Medicine, 331, 244-249. http://dx.doi.org/10.1056/NEJM199407283310407

[45] Lukassen, H.G., Schonbeck, Y., Adang, E.M., Braat, D.D., Zielhuis, G.A. and Kremer, J.A. (2004) Cost Analysis of Singleton versus Twin Pregnancies after in Vitro Fertilization. Fertility and Sterility, 81, 1240-1246. http://dx.doi.org/10.1016/j.fertnstert.2003.10.029

[46] Practice Committee of American Society for Reproductive Medicine (2012) Elective Single-Embryo Transfer. Fertility and Sterility, 97, 835-842. http://dx.doi.org/10.1016/j.fertnstert.2011.11.050

[47] Steinberg, M.L., Boulet, S., Kissin, D., Warner, L. and Jamieson, D.J. (2013) Elective Single Embryo Transfer Trends and Predictors of a Good Perinatal Outcome-United States, 1999 to 2010. Fertility and Sterility, 99, 1937-1943. http://dx.doi.org/10.1016/j.fertnstert.2013.01.134

[48] Gerris, J., De Sutter, P., De Neubourg, D., Van Royen, E., Vander Elst, J., Mangelschots, K., et al. (2004) A Real-Life Prospective Health Economic Study of Elective Single Embryo Transfer versus Two-Embryo Transfer in First IVF/ ICSI Cycles. Human Reproduction, 19, 917-923. http://dx.doi.org/10.1093/humrep/deh188

[49] Wu, A.K., Odisho, A.Y., Washington III, S.L., Katz, P.P. and Smith, J.F. (2014) Out-of-Pocket Fertility Patient Expense: Data from a Multicenter Prospective Infertility Cohort. The Journal of Urology, 191, 427-432. 
http://dx.doi.org/10.1016/j.juro.2013.08.083

[50] Katz, P., Showstack, J., Smith, J.F., Nachtigall, R.D., Millstein, S.G., Wing, H., et al. (2011) Costs of Infertility Treatment: Results from an 18-Month Prospective Cohort Study. Fertility and Sterility, 95, 915-921. http://dx.doi.org/10.1016/j.fertnstert.2010.11.026

[51] Vitek, W.S., Galarraga, O., Klatsky, P.C., Robins, J.C., Carson, S.A. and Blazar, A.S. (2013) Management of the First in Vitro Fertilization Cycle for Unexplained Infertility: A Cost-Effectiveness Analysis of Split in Vitro FertilizationIntracytoplasmic Sperm Injection. Fertility and Sterility, 100, 1381-1388. http://dx.doi.org/10.1016/j.fertnstert.2013.06.035

[52] Grow, D., Kawwass, J.F., Kulkarni, A.D., Durant, T., Jamieson, D.J. and Macaluso, M. (2014) GnRH Agonist and GnRH Antagonist Protocols: Comparison of Outcomes among Good-Prognosis Patients Using National Surveillance Data. Reproductive Biomedicine Online, 29, 299-304. http://dx.doi.org/10.1016/j.rbmo.2014.05.007

[53] Sills, E.S., Collins, G.S., Salem, S.A., Jones, C.A., Peck, A.C. and Salem, R.D. (2012) Balancing Selected Medication Costs with Total Number of Daily Injections: A Preference Analysis of GnRH-Agonist and Antagonist Protocols by IVF Patients. Reproductive Biology and Endocrinology, 10, 67. http://dx.doi.org/10.1186/1477-7827-10-67 